\title{
A HeRAnÇA Da Língua italiana na FALA E NA CULTURA DOS ARGENTINOS: ITALIANISMOS E ITALIANIDADE
}

\author{
Olga Alejandra Mordente*
}

RESUMO: O presente trabalho se propõe a ilustrar de que forma a cultura e a língua sofreram na Argentina significativas mudanças devido à forte imigração italiana. Normalmente são os imigrantes que se adaptam à cultura existente, mas, neste caso, o processo foi inverso ou recíproco. Nosso objetivo principal é, portanto, o de apresentar uma visão geral, por meio de depoimentos e entrevistas, das razões pelas quais uma pessoa de origem italiana decide estudar a língua dos pais ou dos avós, considerando que de 15 a 20 milhões de argentinos têm algum antepassado italiano, ou seja, praticamente metade da população. Queremos também mostrar como os descendentes não revelam, em sua maioria, traços de uma identidade italiana, mas, ao contrário, um interesse em obter a cidadania mais ligado às vantagens e às facilidades que isso representa para os cidadãos argentinos.

PALAVRAS-CHAVE: Língua de herança, imigrantes italianos, descendentes, entrevistas.

ABSTRACT: Questo lavoro ha lo scopo di illustrare come la cultura e la lingua abbiano subito cambiamenti significativi in Argentina a causa della forte immigrazione italiana. Di solito sono gli immigrati che si adattano alla cultura esistente, ma in questo caso il processo è stato inverso o reciproco. Il nostro obiettivo principale è quindi quello di presentare una panoramica, ottenuta attraverso testimonianze e interviste, dei motivi per i quali una persona di origine italiana decide di studiare la lingua dei propri genitori o nonni, considerando che da 15 a 20 milioni di argentini hanno origini italiane, cosa che rappresenta praticamente metà della popolazione. Ci proponiamo di mostrare come i discendenti non rivelino, nella maggior parte dei casi, tracce di un'identità italiana ma, al contrario, un interesse nei confronti della cittadinanza più legato ai vantaggi che ciò rappresenta per i cittadini argentini.

PAROLE CHIAVE: Lingua d'eredità, immigrati italiani, discendenti, interviste.

* Universidade de São Paulo (USP)

alemordente@usp.br

DOI: http://dx.doi.org/10.11606/issn.2238-8281.v0i38p99-111 
ABSTRACT: This work aims to illustrate how culture and language have undergone significant changes in Argentina due to the strong Italian immigration. Usually it is immigrants who adapt to the existing culture, but, in this case, the process was reversed or reciprocal. Our main purpose is, therefore, to present an overview, through testimonials and interviews, of the reasons why a person of Italian origin decides to study the language of their parents or grandparents, considering that 15 to 20 million of Argentines have an Italian ancestor, that is practically half of the population. We want to show how the descendants do not reveal, mostly, traces of an Italian identity, but, on the contrary, an interest in obtaining citizenship more linked to the advantages and facilities that this represents for Argentine citizens.

KEYWORDS: Language of inheritance, Italian immigrants, descendants, interviews. 


\section{Introdução}

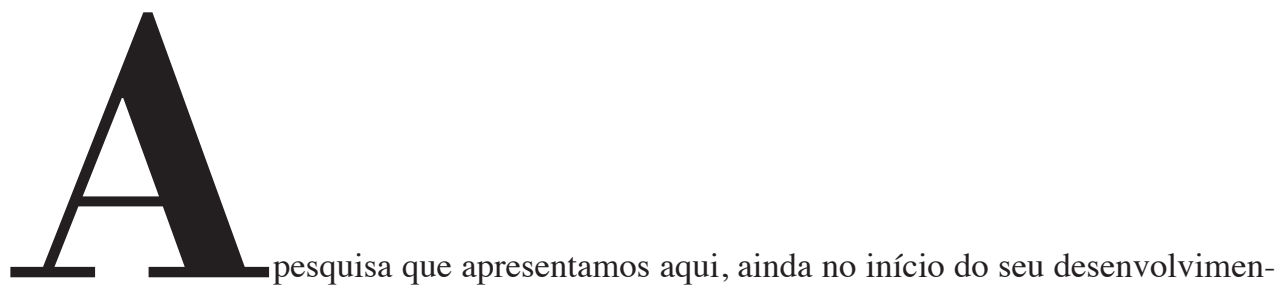

to, constitui-se em um estudo acerca da significativa mudança que a cultura e a língua argentina tiveram devido à forte imigração italiana. $\mathrm{O}$ trabalho justifica-se por contribuir com as reflexões sobre as línguas em contato, especificamente no que diz respeito à língua italiana na fala dos argentinos.

A pesquisa tomou dois rumos: por um lado, foram observados os italianismos que se difundiram rapidamente e circulam junto com muitas palavras de origem italiana adotadas pelos argentinos, mostrando a influência da língua italiana no país; por outro lado, foram investigadas as razões pelas quais os argentinos estudam italiano, buscando entender se há elementos que caracterizariam uma "língua de herança" (SOARES, 2012) e se existem analogias em relação às motivações que levam os brasileiros a estudar o idioma.

\section{A imigração italiana na Argentina e a língua: cocoliche e lunfardo.}

A imigração procedente da Itália foi, de fato, notável na Argentina: em 1885, mais de 12\% da população era constituída por italianos que eram denominados "tanos", de napolitanos. Entre 1857 e 1940, aproximadamente 3 milhões de italianos, cerca de $45 \%$ do total dos estrangeiros imigrantes, chegaram à Argentina.

Considerando que a população argentina é hoje de 44 milhões $^{1}, 15$ a 20 milhões de argentinos têm algum antepassado italiano, ou seja, praticamente metade da população. O fluxo migratório durou até os anos que seguiram a Segunda Guerra Mundial e a maioria dos imigrantes tinha baixa escolarização, o que favoreceu, como veremos com mais detalhes a seguir, o surgimento de uma língua de contato: o chamado cocoliche, que é definido como uma mistura de italiano padrão e de vários dialetos com o espanhol. Falado no passado por imigrantes italianos, foi usado no teatro popular argentino como um elemento cômico e dramático.

Fontanella De Weinberg (1979, p.75), apresentando o cocoliche, afirma que os imigrantes 
italianos, em sua passagem de uma língua para outra, usaram uma variedade de formas intermediárias, cujo desenvolvimento, de acordo com o autor, foi estimulado pelo caráter dialetal na fala de quase todos os imigrantes peninsulares e pelo desconhecimento do italiano padrão (DE WEINBERG, 1979, p.76). Assim, muitos italianos precisavam de uma língua para se comunicar com os argentinos, mas também uns com os outros, pois havia, inclusive, diferenças muito grandes entre os vários dialetos que poderiam impedir a compreensão mútua entre os próprios italianos. Nota-se que o fenômeno do cocoliche foi, portanto, favorecido pela baixa ou ausente escolarização da maioria dos italianos e que esse fenômeno não se registra em italianos com um nível mais alto de educação, pois apenas aqueles com um baixo nível de escolarização, em sua tentativa de se comunicar em espanhol, acabavam utilizando palavras que eram essencialmente de origem dialetal (DE WEINBERG, 1979, p.77).

Uma característica peculiar do cocoliche é sua entoação "italiana" no castelhano, com a mutação do fonema [g] em [k], como em amigo/amico, e a pronúncia do "c" do espanhol antes de vogal que adquire o som "ch", como em dice /diche.

Além do cocoliche, formou-se também o dito lunfardo, que o dicionário da fala dos argentinos (2008, p. 416) define assim: "gergo" empregado originariamente em Buenos Aires por imigrantes marginais e mal viventes. Sobre o lunfardo se diz também que se trata de uma linguagem popular do Rio de la Plata, derivada principalmente da imigração européia, especialmente a italiana, que ocorreu entre os anos de 1870 e 1960 e que emprestou muitas palavras a letras de tango.

Portanto, enquanto o cocoliche representa a maneira de falar espanhol dos imigrantes italianos com baixo nível de instrução, que chegaram na Argentina nas últimas décadas do século XIX e na primeira parte do século XX, o lunfardo se constitui como língua de uma parte da população. Segundo a Secretaria de Cultura da Nação, existem 6.000 termos em lunfardo e Teruggi (1974, p. 26) o considerou como uma fala popular argentina, que surge como consequência da imigração e usa palavras e expressões de outras línguas, especialmente de palavras dialetais italianas, que não são registradas nos dicionários castelhanos. Fonética e entonação, sintaxe e léxico revelam a marca dos dialetos italianos. Nas palavras de Teruggi (1974, p. 21) “o lunfardo não é uma língua ou um idioma, mas um conjunto de palavras e expressões coloquiais", que derivam essencialmente de cinco idiomas: espanhol, italiano, francês, inglês e línguas do grupo galego-português.

\section{Os italianismos na Argentina}

Existem estudos de vários pesquisadores sobre os italianismos em uso hoje em dia na cidade de Buenos Aires (BIHAN, 2011; ZILO, 1970; GOBELLO, 1977). Nossa intenção não é retomar esses estudos, mas mostrar em que medida os italianismos são conhecidos pelos argentinos na sua própria língua e se eles sabem seu significado. Os italianismos foram introduzidos desde os anos 1920 por meio do teatro, das canções e do jornalismo popular que os converteram em uma 
linguagem literária viva e divertida. Gobello e Olivieri realizaram, em 2001, um estudo sobre a evolução do lunfardo até aquele momento e descobriram que não só não desapareceu, mas também foi inserido, por exemplo, no chamado rock crioulo. Elementos que provêm do italiano foram introduzidos, como já mencionamos descrevendo o lunfardo, também em algumas letras de tango. Vejamos a seguir alguns exemplos de palavras procedentes do italiano, de variedades de italiano ou ainda de suas formas dialetais:

(1) ATENTI: “cuidado", “atenção". Segundo Meo Zilio (1970, p. 4), a palavra deriva do ital. attento e se espalhou particularmente pelo tango “Atenti, pebeta”, escrito em 1932 por Flores, Celedonio.

(2) PEBETA: “jovem” (GUARNIERI, 1967, p. 163); do genovês: pivetta, "graciosa jovem".

(4) MINA: "mulher", do italiano femmina.

(5) PIBE: "jovem", do genovês pive no sentido de estagiário.

(6) FIACA: "preguiça", do italiano fiacco, débil, o fiacca, preguiça, falta de vontade, lentidão.

Meo Zilio (1970) aponta que a maior parte dos italianismos chegados ao "Rio del Plata" são de tipo lexical e que procedem do italiano dito standard, do genovês e dos dialetos meridionais e setentrionais, como mostram os exemplos acima citados. Essas palavras são vocábulos do lunfardo e representam parte de um repertório trazido a Buenos Aires pelos imigrantes italianos e incorporado na fala popular da cidade, embora os italianismos tenham passado logo a fazer parte também da fala dos jovens argentinos nativos.

Vejamos outros exemplos:

(7) CUCHA: (lunfardo) "casita del perro", do ital. cuccia (GOBELLO, 1977, p. 55).

(8) COMPARSA: do ital. merid. cumpárza; (lunfardo) 'comparsa', 'grupo de personas vestidas de cierto modo que asisten en corporación a las fiestas de carnaval' [grupo de pessoas vestidas de uma determinada maneira que assistem conjuntamente às festas de carnaval] (GOBELLO, 1977, p.57).

(9) MATINA: "manhã”, do ital. mattina.

(10) CHAO: "adeus", do ital. ciao.

(11) CREPAR: "morrer", do ital. crepare. 
As palavras de origem italiana são muitas e há um grande número em uso na fala cotidiana dos argentinos, sem que eles percebam que são palavras de origem italiana, pois algumas delas já são definitivamente parte do espanhol da Argentina.

Essas premissas sobre uso dos italianismos na fala dos argentinos e nas diversas manifestações culturais são importantes para pensarmos como os argentinos conviveram com línguas de contato, sendo o italiano uma dessas línguas há mais de 100 anos. Alia-se a isso o fato de que esses italianismos foram incorporados na fala argentina, sem que a população estivesse ciente de sua origem linguística. Tendo em vista esse quadro, o italiano na Argentina pode ser considerado até mais de "língua de herança", separada do cotidiano da fala. Ensinar italiano na Argentina precisaria, portanto, colocar o foco nessa convivência constante dos argentinos com os italianismos, levando em conta quanto a propagação da língua italiana na Argentina está ligada a uma questão de identidade.

\section{Os descendentes de imigrantes italianos na Argentina de hoje: motivações para o estudo da língua}

A imigração europeia para a Argentina entre 1880 e 1930, maciça e prolongada no tempo, indica que a Argentina foi percebida como um país de oportunidades, trabalho e crescimento econômico.

Como fizeram seus ancestrais cem anos antes ou mais, muitos argentinos agora colocam suas esperanças na emigração, como um caminho para um futuro que lhes permitirá superar a atual falta de horizontes. Há cem anos, tudo o que era necessário para tentar fazer fortuna na Argentina era ter entre 10 e 59 anos e ser saudável. Hoje, essas duas condições não são suficientes para garantir a um imigrante argentino na Europa o direito de se candidatar a um emprego. Por essa razão, muitos descendentes de europeus na Argentina pedem o reconhecimento de uma cidadania herdada de seus antepassados e recorrem à base de dados de imigrantes em busca de informações precisas sobre a chegada e a origem de seus antepassados europeus.

A população nascida na Itália residente na Argentina está desaparecendo, ficando os descendentes de italianos: filhos, netos e bisnetos.

Os falantes que poderiam ser denominados falantes de herança apresentam características específicas que os distanciam tanto dos falantes nativos monolíngues como dos falantes de uma segunda língua (GUBITOSI, 2012). Os falantes de herança, em geral, utilizam a língua no contexto do lar, da família e, às vezes, com os amigos. Isso implica desconhecimento do léxico necessário para interagir em diferentes registros e estilos, por exemplo, na escola ou em ambientes mais formais. Ao mesmo tempo, verifica-se muitas vezes também a falta de uma competência pragmática em situações formais, mais distantes do âmbito familiar. Em poucas palavras, o insumo que recebem os falantes de uma língua que chamaremos de herança (LH) é restrito, o que obviamente não ocorre com falantes nativos. Por outro lado, cabe lembrar que os falantes das línguas de herança têm uma forte ligação emotiva com a língua dos seus antepassados, diferente do acontece com uma segunda língua, pois os aprendizes, em geral, não possuem 
conexões culturais ou sentimentos de ligação em relação à língua que aprendem.

Algumas das características mais visíveis dos falantes de línguas de herança são:

1. O vocabulário utilizado se restringe ao contexto familiar, com ausência de léxico referentes aos domínios públicos;

2. Empréstimos lexicais são da língua dominante;

3. Há alternância de código entre a língua de herança e a língua dominante;

4. Observam-se transferências da língua hegemônica à língua minoritária.

Nesse contexto, para a pesquisa sobre as motivações que levam os argentinos a estudar italiano, foi aplicado um questionário em italiano que tinha como objetivo traçar o perfil dos aprendizes (v. Anexo 1). O questionário foi também aplicado para saber qual seria a motivação de estudo do italiano, sem levar em conta a origem dos estudantes.

Responderam ao questionário 75 alunos da escola Dante Alighieri Tigre de Buenos Aires, que possuem nível de escolaridade e faixa etária diversificada, sendo 14 anos a idade mínima para se frequentar os cursos para adultos.

A idade dos alunos que participaram da pesquisa variava de 20 a 70 anos. O grupo era composto majoritariamente por mulheres, o nível médio de conhecimento da língua italiano era o B1, segundo o Quadro Europeu Comum de Referência para as línguas (QECR).

Em uma tabela foram registrados os resultados das respostas aos questionários, colocando em primeiro lugar no estudo a origem dos participantes, o motivo do estudo da língua (motivação intrínseca e motivação extrínseca) e as motivações secundárias.

Por meio do questionário distribuído, foi possível determinar por que os argentinos escolhem o italiano como língua estrangeira ou de herança. Os cursos em que foram distribuídos os questionários não eram específicos para descendentes de italianos e não poderiam, portanto, ser definido de LH: no mesmo grupo, existiam interesses diferentes e o estudo do italiano como língua estrangeira tinha as mais diversas razões, sendo que havia entre elas o contato com o italiano para cultivar a língua de origem.

No questionário todos os envolvidos responderam livremente em italiano e obtivemos os seguintes dados (a versão completa dos resultados está no Anexo 2) sobre os motivos que levam ao estudo da língua italiana:

(a) Participantes de 20-30 anos: estudam italiano porque gostam da língua e da cultura italiana e consideram importante saber outro idioma. Não se percebem sinais que possam remeter a uma identidade italiana.

(b) Participantes de 40-50 anos: o interesse em aprender o italiano deve-se não somente ao fato que os aprendizes gostam da língua, mas também à vontade de conhecer a língua de sua ascendência. Entre as motivações, são mencionadas também as seguintes: para poder ler um livro, um jornal ou escutar uma canção e entender sua letra.

(c) Participantes da 60-70 anos: o interesse principal é conhecer as suas raízes. Além disso, são citados: a musicalidade do idioma, o prazer, o fato de poder comunicar-se com a família na Itália, ler textos literários ou viajar para Itália, a tradição cultural, literária e artística 
da Itália. Todos os participantes que responderam têm origem italiana. A maioria tem algum parente que fala italiano.

\section{Primeiras conclusões}

Por mais que o questionário tenha sido aplicado a um número relativamente pequeno de estudantes e ainda que tenha sido utilizado em cursos diferentes, pudemos chegar a algumas conclusões preliminares. Primeiramente, todos os entrevistados são de origem italiana e têm algum parente que fala ou falou italiano na família. O motivo do estudo da língua deve-se essencialmente, entre os mais jovens, ao interesse em conhecer outro idioma, ao gosto pela língua e pela cultura italiana, ao desejo de comunicar-se com pessoas que falam italiano e de viajar comunicando-se melhor. Entre esses participantes, não se percebem sinais de identidade italiana, mas é evidente o interesse pela obtenção de um passaporte italiano, que, por ser da União Europeia, é valorizado porque permite ingressar em qualquer país da Europa e do mundo. Entre os participantes de faixa etária entre 60 e 75 anos, muitos fazem afirmações que se poderiam resumir da seguinte maneira: "Quero a cidadania não para mim porque eu tenho minha profissão, mas para o futuro de meus filhos e meus netos".

À medida que a idade aumenta, as razões que as razões que levam a querer estudar a língua italiana ou aproximar-se da cultura diferem bastante. Os participantes entre 40 e 50 anos estudam italiano ou procuram um curso, sobretudo, para saber a língua dos seus antepassados ou para poder ler um livro ou jornal italiano ou compreender o que diz uma canção.

Os participantes entre 60 e 70 anos estudam a língua para aprimorá-la, para poder viajar para a Itália, para rever ou procurar seus parentes italianos ou para conseguir ler textos literários italianos.

O que podemos verificar é, portanto, que entre os mais jovens os traços de italianidade diminuem, como demonstra o fato de eles se interessarem pelo estudo da língua italiana assim como se interessariam por qualquer outro idioma, embora possa ser talvez reconhecida a presença de uma certa tradição que não é, no entanto, "italianidade".

Os argentinos da capital e de algumas províncias argentinas sempre conviveram com línguas de contato, embora em diferentes graus. Por isso, para eles, as palavras de origem italiana ou dialetal, que fazem parte do repertório linguístico do castelhano da Argentina, são compreendidas muito bem tanto que os argentinos não percebem que não são palavras ou expressões de sua própria língua. É muito conhecida uma frase de Jorge Luís Borges que remete a essa dimensão pluricultural e plurilinguística dos argentinos: "O argentino é um italiano que fala espanhol, pensa em francês e gostaria de ser inglês".

Os dados coletados até agora mostram que os jovens argentinos de hoje são talvez menos "italianos", o que se explica pelo natural distanciamento dos antepassados que imigraram na Argentina. Mesmo assim, a Itália ainda está lá. A continuação da nossa pesquisa poderá nos ajudar a trazer mais dados e detalhes sobre isso. 


\section{Referências}

DE WEIMBERG, M.B.F. La asimilación lingüística de los inmigrantes. Bahía Blanca: Departamento De Ciencias Sociales Universidad Nacional Del Sur, 1979.

GOBELLO, J. (1977) Diccionario Lunfardo y de otros términos antiguos y modernos usuales en Buenos Aires. Buenos Aires: A. Peña Lillo Editor S. R. L, 1977.

.\& Oliveri, M.H. Tangueces y lunfardismos del rock argentino. Buenos Aires: Corregidor, 2001.

GUBITOSI, P. El desafio de enseñar lengua a los hablantes de herencia. In: Identidades dinámicas: variación y cambio en el español de América. La Plata, 2012. Disponível em: http:// jornadasfilologiaylinguistica.fahce.unlp.edu.ar.

GUARNIERI, J. C. El habla del lenguaje del boliche: diccionario popular rioplatense. Montevideo: Editorial Florensa \& Lafon, 1967.

LE BIHAN, U. Italianismos en el habla de la Argentina: herencia de la inmigración italiana. Cocoliche y lunfardo. Universidade de Oslo, Master, 2011. Disponível em: http://www.duo.uio.no.

MEO ZILIO, G. El elemento italiano en el habla de Buenos Aires y Montevideo. Firenze: Valmartina Editore, 1970.

SOARES CAMPOS DUARTE, S.M. de C. Português Língua de Herança: da Teoria à Prática. Dissertação. 2012. Faculdade de Letras - Universidade do Porto.

TERUGGI, M.E. Panorama del lunfardo. Buenos Aires: Ediciones Cabargon, 1974.

Recebido em: 04/12/2019

Aprovado em: 28/12/2019 


\section{ANEXO 1 - QUESTIONÁRIO UTILIZADO NA PESQUISA}

1. Quanti anni hai?

2. Quali sono i cognomi dei tuoi nonni?

3. Qualcuno della tua famiglia parla italiano? Chi?

4. Perché vuoi studiare la lingua italiana?

La motivazione intrinseca è la tua motivazione e include i tuoi obiettivi, i tuoi valori e i tuoi interessi

a) Scrivi tre motivi per cui vuoi imparare la lingua italiana: concentrati sui tuoi bisogni, le tue curiosità, il tuo piacere:

1.

2.

3.

La motivazione estrinseca viene da fuori ed è meno efficace della motivazione intrinseca. Comprende gli obiettivi e gli interessi di altri e come questi ti influenzano. Esempi:

○ Imparo l’italiano perché lo richiede il lavoro.

${ }^{\circ}$ Imparo l'italiano per superare un test. 
b) Scrivi tre ragioni per cui qualcun altro vuole che tu impari la lingua italiana:

1.

2.

3.

c) Ora segna con una crocetta le sottomotivazioni per lo studio dell'italiano che ti riguardano. Oltre alla crocetta, indica, per favore, un ordine di priorità $(1,2,3 \ldots)$

Seguire spettacoli in italiano

Leggere testi letterari

Seguire la radiotelevisione italiana

Leggere testi scientifici in italiano

Laurearsi in Italianistica

Seguire un corso di perfezionamento in Italia

Lavorare in rapporto con l'Italia

Rapporti familiari in Italia

Interesse per la cultura italiana

Rapporti d'amicizia in Italia.

Grazie! 
ANEXO 2

TABELLA DEI RISULTATI (QUADRO COM OS RESULTADOS)

\begin{tabular}{|c|c|c|c|}
\hline $\begin{array}{l}\text { FASCIA } \\
\text { D'ETÀ }\end{array}$ & $20-30$ & $40-50$ & $60-70$ \\
\hline Origine & Italiana & Italiana & Italiana \\
\hline $\begin{array}{l}\text { Qualcuno della } \\
\text { famiglia parla } \\
\text { italiano }\end{array}$ & $\begin{array}{l}\text { Nessuno/i genitori, i } \\
\text { nonni e le zie. }\end{array}$ & $\begin{array}{l}\text { I miei nonni, mio } \\
\text { padre, mia madre }\end{array}$ & Mio nonno \\
\hline $\begin{array}{l}\text { Motivazioni } \\
\text { per lo studio } \\
\text { dell'italiano }\end{array}$ & $\begin{array}{l}\square \quad \text { Mi piacciono } \\
\text { la lingua e la cultura } \\
\text { italiana } \\
\square \quad \text { Mi piace } \\
\text { sapere un'altra lingua }\end{array}$ & $\begin{array}{l}\square \quad \text { Mi piace la } \\
\text { lingua del paese } \\
\square \quad \text { Per sapere la } \\
\text { lingua dei miei ante- } \\
\text { nati }\end{array}$ & $\begin{array}{l}\square \quad \text { Per piacere } \\
\square \quad \text { Per conoscere } \\
\text { le loro radici } \\
\square \quad \text { Mi piace leg- } \\
\text { gere scrittori italiani }\end{array}$ \\
\hline $\begin{array}{l}\text { Motivazione } \\
\text { intrinseca }\end{array}$ & $\begin{array}{l}\square \quad \text { Volere comu- } \\
\text { nicarsi con persone } \\
\text { che parlano l'italiano } \\
\square \quad \text { Apprendere } \\
\text { un altro idioma e } \\
\text { un'altra cultura } \\
\square \quad \text { Accedere a } \\
\text { libri, film, e musica. }\end{array}$ & $\begin{array}{l}\square \quad \text { Per conoscere } \\
\text { la cultura } \\
\square \quad \text { È utile se si } \\
\text { vuole viaggiare } \\
\square \quad \text { Mi piace la } \\
\text { musica italiana } \\
\square \quad \text { Per sapere la } \\
\text { lingua degli antenati } \\
\text { italiani } \quad \text { Per poter } \\
\square \quad \text { Per poter } \\
\text { leggere un libro, un } \\
\text { quotidiano italiano, } \\
\square \quad \text { ascoltare e sapere } \\
\text { quello che dice una } \\
\text { canzone }\end{array}$ & $\begin{array}{l}\square \quad \text { Per coprire } \\
\text { altre culture } \\
\square \quad \text { Per poter } \\
\text { comunicare } \\
\square \quad \text { Poter fare un } \\
\text { viaggio in Italia } \\
\square \quad \text { Per onore ai } \\
\text { nonni } \quad \\
\square \quad \text { Per comu- } \\
\text { nicare con gli amici } \\
\text { italiani e i familiari in } \\
\text { Italia }\end{array}$ \\
\hline
\end{tabular}




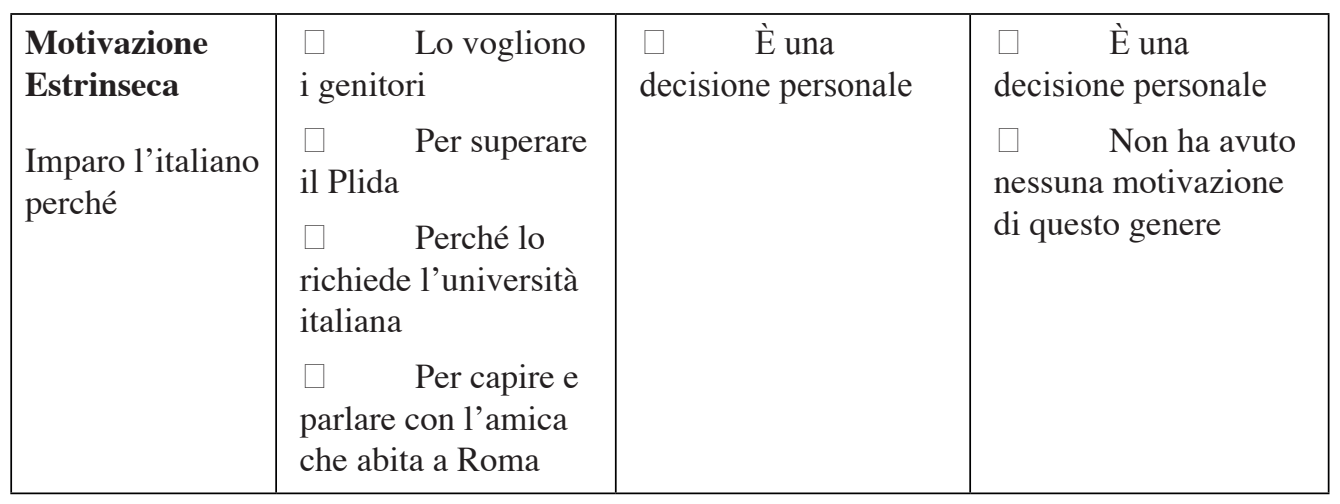

\begin{tabular}{|c|c|c|c|}
\hline $\begin{array}{l}\text { Sottomotiva- } \\
\text { zioni }\end{array}$ & $\begin{array}{l}\square \quad \text { Interesse per } \\
\text { la cultura italiana }\end{array}$ & $\begin{array}{l}\square \quad \text { Interesse per } \\
\text { la cultura italiana }\end{array}$ & $\begin{array}{l}\square \quad \text { Per leggere } \\
\text { testi letterari }\end{array}$ \\
\hline \multirow{3}{*}{$\begin{array}{l}\text { per lo studio } \\
\text { dell'italiano }\end{array}$} & $\begin{array}{l}\square \quad \text { Per seguire } \\
\text { un corso in Italia }\end{array}$ & $\begin{array}{l}\square \quad \text { Per leggere } \\
\text { testi letterari }\end{array}$ & $\begin{array}{l}\square \quad \text { Per i rapporti } \\
\text { familiari in Italia }\end{array}$ \\
\hline & $\begin{array}{l}\square \quad \text { Per leggere } \\
\text { testi scientifici in } \\
\text { italiano }\end{array}$ & $\begin{array}{l}\square \quad \text { Per lavorare } \\
\text { con l'Italia }\end{array}$ & $\begin{array}{l}\square \quad \text { Per i rapporti } \\
\text { d'amicizia in Italia } \\
\text { interesse per la cultura }\end{array}$ \\
\hline & $\begin{array}{l}\square \quad \text { Per seguire } \\
\text { spettacoli in italiano }\end{array}$ & & and \\
\hline
\end{tabular}

